\title{
Erratum to: Medical Imaging and Augmented Reality
}

\author{
Guoyan Zheng ${ }^{1(\bowtie)}$, Hongen Liao $^{2}$, Pierre Jannin ${ }^{3}$, Philippe Cattin ${ }^{4}$, \\ and Su-Lin Lee ${ }^{5}$ \\ ${ }^{1}$ Institute for Surgical Technology, University of Bern, Bern, Switzerland \\ guoyan.zheng@istb.unibe.ch \\ 2 Department of Biomedical Engineering, School of Medicine, Tsinghua \\ University, Beijing, China \\ ${ }^{3}$ Faculte de Medicine, Universite de Rennes 1, Rennes Cedex, France \\ ${ }^{4}$ University of Basel, Allschwil, Switzerland \\ ${ }^{5}$ Hamlyn Centre, Imperial College London, London, UK
}

\section{Erratum to:}

G. Zheng et al. (Eds.)

\section{Medical Imaging and Augmented Reality}

DOI: $10.1007 / 978-3-319-43775-0$

The affiliation of Hongen Liao was incorrect.

The correct affiliation should read as below:

Hongen Liao

Tsinghua University

Department of Biomedical Engineering

School of Medicine

Beijing, China 